\title{
Organogenesis and distribution of the ocular lymphatic vessels in the anterior eye
}

Yifan Wu, ${ }^{1,2}$ Young Jin Seong, ${ }^{1,2}$ Kin Li, ${ }^{1,2,3}$ Dongwon Choi, ${ }^{1,2}$ Eunkyung Park, ${ }^{1,2}$ George H. Daghlian, ${ }^{1,2}$ Eunson Jung, ${ }^{1,2}$ Khoa Bui, ${ }^{1,2}$ Luping Zhao, ${ }^{1,2}$ Shrimika Madhavan, ${ }^{1,2}$ Saren Daghlian, ,

Patill Daghlian, ${ }^{1,2}$ Desmond Chin, ${ }^{1,2}$ II-Taeg Cho, ${ }^{1,2}$ Alex K. Wong, ${ }^{1}$ Martin Heur, ${ }^{4}$ Sandy Zhang-Nunes, ${ }^{4}$ James C. Tan, ${ }^{5}$ Masatsugu Ema, ${ }^{6}$ Tina T. Wong, ${ }^{7}$ Alex S. Huang, ${ }^{5}$ and Young-Kwon Hong ${ }^{1,2}$

'Department of Surgery and ${ }^{2}$ Department of Biochemistry and Molecular Medicine, Norris Comprehensive Cancer Center, Keck School of Medicine of USC, USC, Los Angeles, California, USA. ${ }^{3}$ College of Osteopathic Medicine of the Pacific, Western University of Health Sciences, Pomona, California, USA. ${ }^{4}$ Department of Ophthalmology, USC Roski Eye Institute, Keck School of Medicine of USC, USC, Los Angeles, California, USA. ${ }^{5}$ Doheny Eye Institute and Department of Ophthalmology, David Geffen School of Medicine at UCLA, UCLA, Los Angeles, California, USA. ${ }^{6}$ Department of Stem Cells and Human Disease Models Research Center for Animal Life, Science Shiga University of Medical Science, Seta Tsukinowacho, Otsu, Shiga, Japan. 'TSingapore Eye Research Institute, Duke NUS Graduate Medical School, Singapore.

Glaucoma surgeries, such as trabeculectomy, are performed to lower intraocular pressure to reduce risk of vision loss. These surgeries create a new passage in the eye that reroutes the aqueous humor outflow to the subconjunctival space, where the fluid is presumably absorbed by the conjunctival lymphatics. Here, we characterized the development and function of the ocular lymphatics using transgenic lymphatic reporter mice and rats. We found that the limbal and conjunctival lymphatic networks are progressively formed from a primary lymphatic vessel that grows from the nasal-side medial canthus region at birth. This primary lymphatic vessel immediately branches out, invades the limbus and conjunctiva, and bidirectionally encircles the cornea. As a result, the distribution of the ocular lymphatics is significantly polarized toward the nasal side, and the limbal lymphatics are directly connected to the conjunctival lymphatics. New lymphatic sprouts are produced mainly from the nasal-side limbal lymphatics, posing the nasal side of the eye as more responsive to fluid drainage and inflammatory stimuli. Consistent with this polarized distribution of the ocular lymphatics, a higher drainage efficiency was observed in the nasal side than the temporal side of the eye when injected with a fluorescent tracer. In contrast, blood vessels are evenly distributed at the anterior surface of the eyes. Also, we found that these distinct vascular distribution patterns were conserved in human eyes. Together, our study demonstrated that the ocular surface lymphatics are more densely present in the nasal side and uncovered the potential clinical benefits in selecting the nasal side as a glaucoma surgery site to improve fluid drainage.

Conflict of interest: ASH discloses the following positions and supports: Santen Pharmaceutical (consultant); Allergan, AbbVie (consultant); Aeries Pharmaceuticals (consultant); Heidelberg Engineering (research support); Claukos Corporation (research support); and Diagnosys (research support).

Copyright: (ㄷ) 2020, American Society for Clinical Investigation.

Submitted: December 3, 2019

Accepted: May 27, 2020

Published: July $9,2020$.

Reference information: /CI Insight. 2020;5(13):e135121.

https://doi.org/10.1172/jici.

insight.135121. 
the anterior chamber of the eye. This specialized vascular structure is lined by a single layer of endothelial cells that displays features of both blood vascular endothelial cells (BECs) and lymphatic endothelial cells (LECs). The inner wall of Schlemm's canal is attached to the trabecular meshwork (TM), a region capable of modulating drainage rate by controlled contractions. Like BECs and LECs, Schlemm's canal endothelial cells express several endothelial cell markers, such as CD31, cadherin 5, VEGF receptor 2, and vWF. We and others reported that Schlemm's canal endothelial cells express the master regulator of lymphatics, prospero homeobox 1 (Prox1), which plays a crucial role in Schlemm's canal development (3-7).

The ocular lymphatics and Schlemm's canal share significant similarities in their organogenesis. For lymphatic vessels, a subset of venous endothelial cells upregulate Prox1 and undergo lymphatic differentiation by upregulating LEC signature genes and downregulating BEC-specific genes $(8,9)$. These Prox $1^{+}$lymphatic precursor cells migrate out and form the initial lymphatic vessels. In Schlemm's canal, a subset of limbal BECs begins to express Prox 1 and clusters to form the primitive tube structure, a process termed canalogenesis $(3,4)$. The mature Schlemm's canal remains connected to the blood vessels via collecting channels, which drain the aqueous humor to the systemic circulation. Functionally, both Schlemm's canal and the ocular lymphatics play vital roles in controlling the ocular fluid homeostasis by constituting 2 routes of aqueous humor outflow facilities, termed conventional and nonconventional pathways $(10,11)$. Recently, several studies have also revealed additional roles of the ocular lymphatics in eye health and disease (11-16).

In normal eyes, the TM and Schlemm's canal carefully regulate the degree of outflow resistance to maintain a narrow range of healthy intraocular pressure (IOP) levels. In glaucomatous (open-angle) eyes, however, outflow resistance is often significantly increased, leading to ocular hypertension and optic nerve damage $(17,18)$. In detail, aqueous humor flows from the ciliary processes into the anterior chamber; the aqueous humor then flows into the TM and the inner wall of the Schlemm's canal, before it flows into the systemic circulation via collecting channels $(19,20)$. These regions are thought to contribute to aqueous humor outflow resistance that elevates the $\operatorname{IOP}(19,20)$. While there are numerous pharmacological and laser procedures to alleviate high IOP in glaucoma patients, trabeculectomy is one of the most common surgical procedures if initial interventions fail $(18,21)$. In this procedure, surgeons excise a conjunctival and scleral flap to expose Schlemm's canal and then create a new passage between the scleral layer and the subconjunctival space of the eye. This allows aqueous humor to bypass the point of resistance at the TM and Schlemm's canal $(18,21,22)$. Although conjunctival lymphatics presumably absorb the drained aqueous humor, lymphatic involvement in this process has not been confirmed. Therefore, it is crucial to have a better understanding of the development and function of the conjunctival lymphatic vessels and their role in fluid clearance.

Previous studies have characterized limbal/corneal lymphatics in mice and humans (13, 23-36). Notably, 2 previous pioneering studies by Ecoiffier et al. have demonstrated that the limbal lymphatic vessels are unevenly distributed around the limbal circle in normal and inflamed conditions and have provided robust clinical evidence that the limbal lymphatics play critical roles in corneal inflammation and transplant rejection $(25,37)$. Moreover, these 2 studies have raised additional essential questions as to when and how the ocular lymphangiogenesis occurs to establish the limbal and conjunctival lymphatic networks, as well as the functional significance of the unique ocular lymphatic distribution pattern. In this report, we performed detailed morphological studies on the organogenesis of the ocular lymphatics and Schlemm's canal using potentially novel lymphatic reporter transgenic animals (mouse and rat). We found that the ocular lymphatics enter the corneal limbus from the nasal side, bifurcate, and encircle the cornea, a sequential process that is very different from the development of Schlemm's canal (3, 4). Together, our data presented here improve the current understanding of the ocular lymphatics and Schlemm's canal development, as well as their roles in ocular fluid homeostasis.

\section{Results}

Visualization of the ocular surface lymphatic vessels and Schlemm's canal using transgenic reporter animals. We first characterized the distribution and molecular expression of the lymphatics present in the anterior segment of the postnatal eyes. Because previous reports have clearly documented the expression pattern of Prox1, Cd31, VEGF receptor 3 (Vegfr3), and lymphatic vessel endothelial hyaluronan receptor 1 (Lyve1) in the ocular lymphatics and Schlemm's canal (3-7), we visualized the lymphatic networks in 2 independent bacterial artificial chromosome-based (BAC-based) lymphatic reporter mice that we have previously reported, namely the 
Prox1-EGFP and Prox1-tdTomato transgenic lines $(38,39)$. Consistent with the previous reports, the ocular lymphatics and Schlemm's canal were readily detectable in the Prox1 promoter-driven reporter mice by their EGFP expression (Figure 1, A-C). The ocular lymphatics and Schlemm's canal demonstrated a clear morphological difference: while the limbal and conjunctival lymphatics were relatively thin and branched, Schlemm's canal appeared to be thicker and unbranched. Also, the lymphatics are equipped with frequent luminal valves that express a high level of Prox1 (40), while Schlemm's canal lacks equivalent valves (Figure $1, \mathrm{~B}$ and C). Consistent with previous studies $(3-6,22)$, both the ocular lymphatics and Schlemm's canal expressed Cd31 and Vegfr3, whereas only the ocular lymphatics expressed Lyve1 (Figure 1, D-O) (3-6, 23). The limbal lymphatics are positioned at the outer surface of the limbus, while Schlemm's canal is located at the inner side of the limbus, near the base of the iris (Figure 2A). Moreover, Prox1 was strongly expressed in both the inner and outer walls of Schlemm's canal. It has not been reported whether the limbal lymphatics harbor the luminal valves, a hallmark of collecting lymphatics, and how they extend beyond the limbus area. To address these questions, we performed further studies on the limbal lymphatics using 2 rodent species, Prox1-tdTomato mice (38) and Prox1-EGFP transgenic rats (7). Indeed, we found that the limbal lymphatics are directly connected to the conjunctival lymphatics through multiple short lymphatics present along the corneal edge (Figure 2, B-F). We also detected the Prox $1^{\text {hi }}$ luminal valves in mature limbal lymphatics in adults (Figure 2, C and D), suggesting noncapillary characteristics of limbal lymphatics $(2,41)$. Together, the ocular lymphatics and Schlemm's canal display distinct anatomic and molecular characteristics.

In vivo detection of the ocular lymphatic vessel connection to the extraocular tissues. We next set out to perform in-depth morphological analyses on the ocular lymphatics using Prox1-tdTomato mice (38) and Prox1-EGFP transgenic rats (7). Whole heads of the reporter animals were fixed with paraformaldehyde to preserve the structures of the ocular and surrounding tissues. We found that the limbus and conjunctival tissues, but not the cornea, were richly endowed with the lymphatic networks (Figure 3). Importantly, the lymphatics were more densely distributed in the nasal side than the temporal side, and these ocular lymphatic networks appeared to exit toward the medial canthus of the eye in both species. Enlarged images revealed that a strand of the large-caliber lymphatic vessel comes into the nasal-side conjunctiva from the medial canthus and branches out to form the conjunctival lymphatic networks. Together, the lymphatic reporter mice and rats commonly revealed abundant lymphatic networks in the conjunctiva, which appeared to originate from the medial canthus region.

Stepwise growth of the ocular lymphatics from the nasal side in mouse and rat. The lymphatic distribution shown above prompted us to investigate how the ocular lymphatics postnatally develop and form dense networks in the conjunctiva and limbus. To address this question, we enucleated the developing eyes of the lymphatic reporter newborn pups and adults and prepared corneal flat mounts to visualize the postnatal ocular lymphangiogenesis. We found that a nascent lymphatic vessel originates from the nasal side, infiltrates into the conjunctiva area, bifurcates, and sprouts both clockwise and counterclockwise and eventually covers the limbal and conjunctiva area within the first 2 weeks of postnatal development (Figure 4, A-H). The limbal and conjunctival lymphatics not only concurrently encircled the cornea in parallel but also established intervascular connections using short perpendicular branches en route (Figure 2, B-E). In comparison, Schlemm's canal independently developed without any connections to the ocular lymphatics and became visible by postnatal days 6-8 (arrows, Figure 4, G and H). Quantitative analyses revealed that ocular (limbal and conjunctival) lymphatic vessels were formed at about 3-4 times higher density in the nasal side as compared with the temporal side, and this polarized lymphatic distribution was maintained in adults (Figure 4, I and J). Intriguingly, we noted that all ocular lymphatics seemed to grow out from 1 (rarely 2) lymphatic trunk in the eye of neonates (P5.5) and that this thick trunk appeared to serve as the root of the entire lymphatic vascular tree (Figure $4 \mathrm{~K}$ ). We also confirmed the nasal-side predilection pattern of the ocular lymphatic development in Prox1-EGFP lymphatic reporter rats (7). Indeed, we observed a comparable pattern of ocular lymphangiogenesis in rats: the limbal and conjunctival lymphatics in newborn rat pups (P2) migrated from the nasal side and bifurcated to encircle the cornea (Figure 4L). Notably, Schlemm's canal had not yet formed at this point. At P9, while the limbal and conjunctival lymphatics are in the process of encircling the cornea, Schlemm's canal developed evenly throughout the corneal edge (Figure 4, M and N). It was interesting to find that the ocular lymphatics development exhibited unidirectional growth from the nasal to temporal side, whereas Schlemm's canal was uniformly established over a few days around P5-P8 in a nonpolarized manner (3-6). In addition, we imaged the lateral view of the eye of Prox1-tdTomato adult mice from different angles and validated the root of the conjunctival lymphatic network in 3-dimensional intact images (Figure 5, A-E). The sequential lateral views of a Prox1-tdTomato 

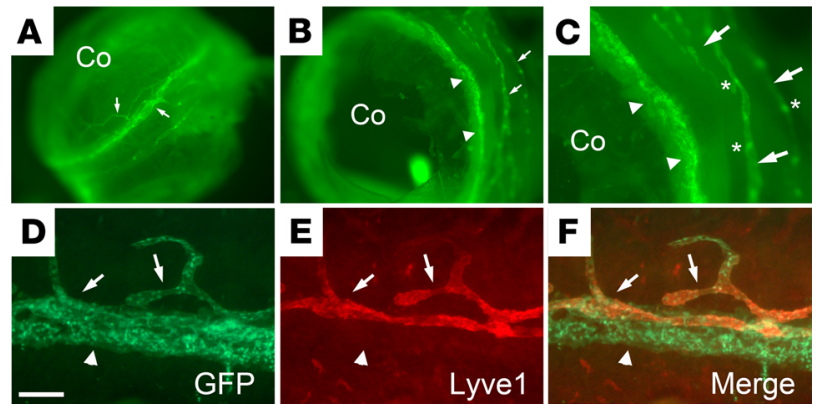

Figure 1. Visualization of the ocular lymphatic vessels and Schlemm's canal. (A-C) Lateral view of the ocular lymphatics (arrows) and Schlemm's canal (arrowheads) in the anterior segment of the eye of a Prox1-EGFP transgenic mouse. The limbus area in panel $\mathbf{B}$ is enlarged 2.3 times in panel $\mathbf{C}$, where lymphatic valves (asterisks) are identified by stronger EGFP (Prox1) expression than luminal LECs. (D-F) Lyve1 whole-mount staining (red) showing the limbal lymphatics and Schlemm's canal in the eye of a Prox1-EGFP mouse. While EGFP (Prox1) is expressed in both Schlemm's canal (arrowhead) and the limbal lymphatics (arrows), Lyve1 is detected only in the limbal lymphatics, not in Schlemm's canal. (G-L) Anti-Cd31 staining (green) of the limbal blood and lymphatic vessels and Schlemm's canal in the eyes of a Prox1-tdTomato reporter mouse. Schlemm's canal (arrowhead) and the limbal lymphatics (arrow), but not
limbal blood vessels (double arrows), are selectively shown by Prox1-tdTomato
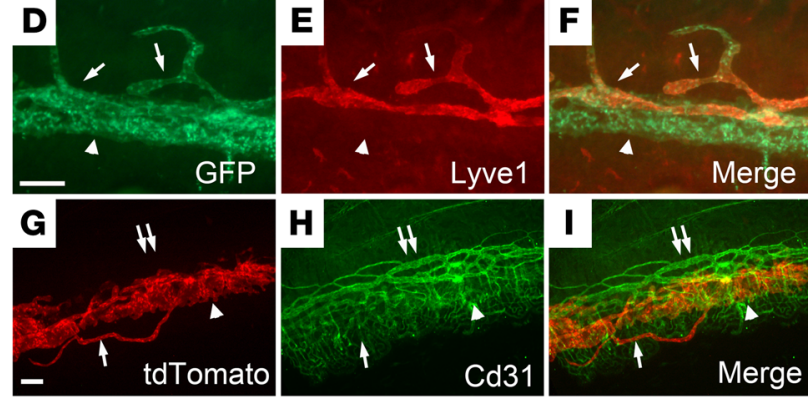
reporter (red). (M-0) Anti-Vegfr3 staining (green) in the eyes of a Prox1-tdTomato reporter mouse. Vegfr 3 is expressed in both the limbal lymphatics (arrow) and Schlemm's canal (arrowhead). Adult mice (6-10 weeks old, both sexes, $n=4$ ) were used for all panels. One representative image is shown for each panel. Co, cornea. Scale bar: $100 \mu \mathrm{m}$.
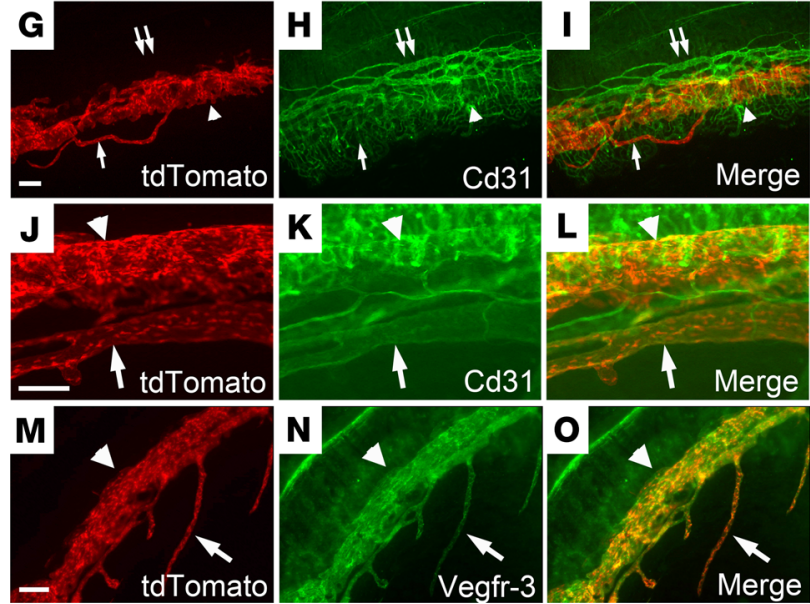

adult eye showed that the entire conjunctival lymphatics are rooted to 1 major collecting vessel (Figure $5 \mathrm{~F}$ ), suggesting that the lymphatic root collector may funnel all lymph fluid taken by the ocular lymphatics to the regional lymph nodes and eventually to the circulation. Together, this study provides detailed information about the developmental timing and morphogenesis of the ocular lymphatic networks.
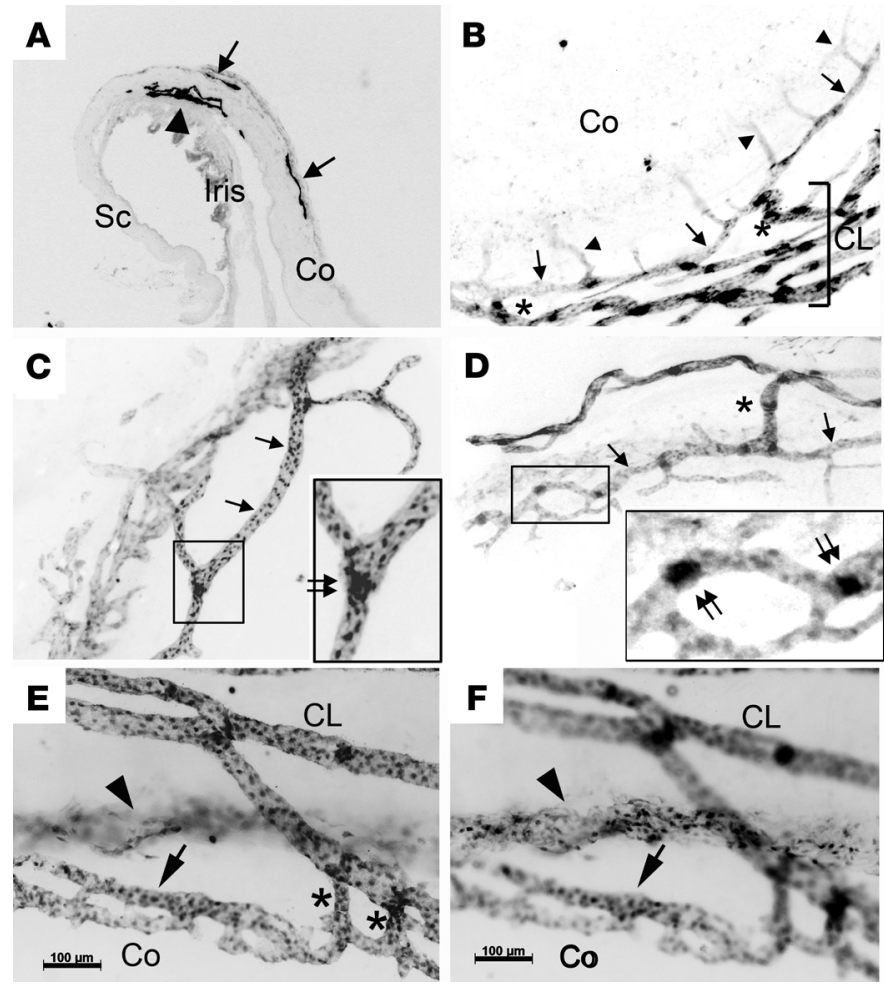

Figure 2. Spatial distribution of the limbal and conjunctival lymphatics and Schlemm's canal. (A) Expression of the reporter (EGFP) in the inner and outer walls of Schlemm's canal (arrowhead) as well as the limbal lymphatics (arrow) in a frozen cross section of the eye of a Prox1-EGFP mouse. (B-D) The limbal lymphatics (arrows) and their sprouts (arrowheads) are equipped with lymphatic valves and directly connected to the conjunctival lymphatics (CL). The connections are marked with asterisks in panels $\mathbf{B}$ and $\mathbf{D}$, and the lymphatic valves (double arrows) are shown in panels $\mathbf{C}$ and $\mathbf{D}$, where the boxed areas are enlarged in the insets (by 2 times in $\mathbf{C}$ and 2.7 times in $\mathbf{D}$ ). Adult mice (6-10 weeks old) were used for panels A-D. (E and F) High-magnification images, focusing on either the lymphatics (E) or Schlemm's canal (F), in the same location of the eyes of a Prox1-EGFP rat (P5). The limbal lymphatics (arrow), Schlemm's canal (arrowhead), and CL are visualized, and the limbal-to-conjunctival lymphatic connection is marked with asterisks. Co, cornea; Sc, sclera. Scale bars: $100 \mu \mathrm{m}$. At least 3 eyes from the reporter animals (both sexes) were used for each experiment, and 1 representative image is shown for each panel. 

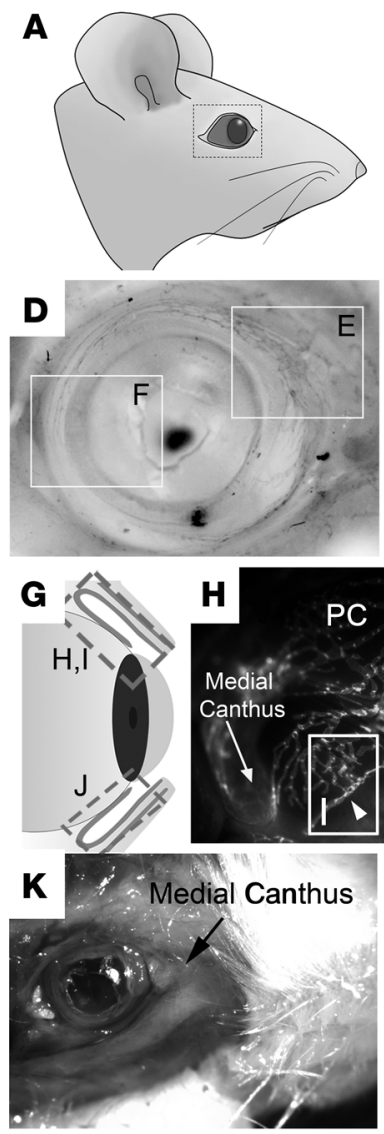
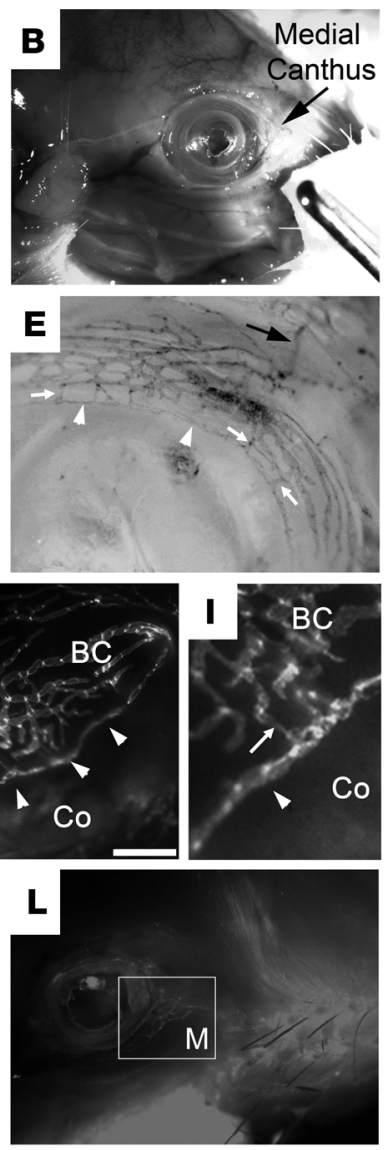
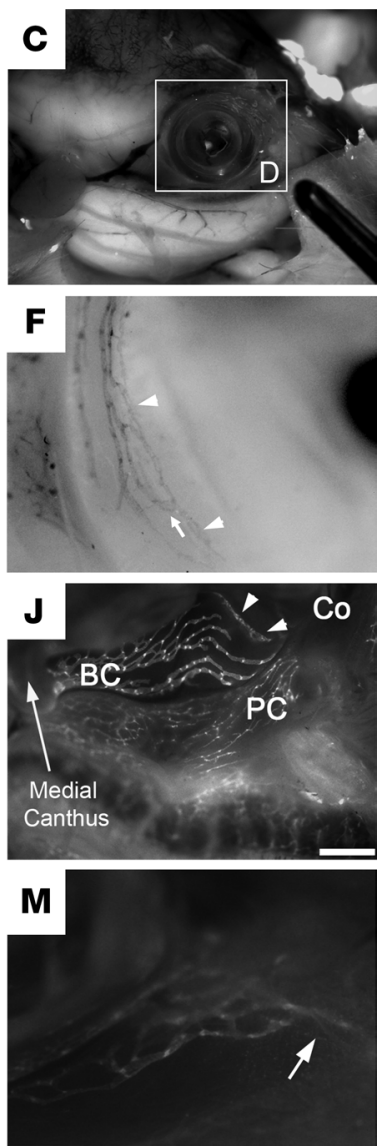

Figure 3. In situ visualization of the lymphatic vessels in the ocular and surrounding tissues. (A-F) The whole head of a Prox1-EGFP rat was fixed and partially dissected to reveal the ocular lymphatics surrounding the ocular tissue. (A) Diagram of the rat head showing the region of interest. Bright-field (B) and fluorescence (C) images of the partially skinned head of a Prox1-EGFP rat. (D) Inverted and gray scale image of the ocular lymphatics with 2 boxed areas that are enlarged in panels $\mathbf{E}$ and $\mathbf{F}$. The limbal lymphatics (white arrowheads) are directly attached to the conjunctival lymphatics through multiple short branches (white arrows). (G-J) Dense lymphatic networks in the conjunctiva. (C) Simplified diagram showing the superior ( $\mathbf{H}$ and $\mathbf{I}$ ) and inferior $(\mathbf{J})$ conjunctiva in the nasal side. (I) The boxed area in panel $\mathbf{H}$ was enlarged. Arrow points to the connection between the conjunctival lymphatics and the limbal lymphatics (arrowheads). PC, palpebral conjunctiva; BC, bulbar conjunctiva; Co, cornea. Scale bars: $1 \mathrm{~mm}$. (K-M) The head of a Prox1-tdTomato mouse was fixed and partially skinned to reveal the lymphatics surrounding the ocular tissue. Arrow in panels $\mathbf{E}$ and $\mathbf{M}$ point to a main lymphatic vessel that exits the ocular tissue and extends toward the medial canthus. Five rats and 7 mice (both sexes) were used for each experiment with comparable results. One representative image is shown for each panel. Original magnification, $\times 2.7(\mathbf{D}, \mathbf{E}$, and $\mathbf{F}), \times 3(\mathbf{I})$, and $\times 3.9(\mathrm{M})$.

More efficient conjunctival drainage in the nasal side than the temporal side. We next asked whether fluid drainage is more efficient in the nasal side than the temporal side because of higher lymphatic density in the nasal-side conjunctiva. To compare the drainage of the 2 sides, we injected a fluorescent tracer (albumin red, MW $=66 \mathrm{kDa}$ ) into the nasal or temporal side of the eyes of Prox1-EGFP mice. Indeed, the tracer injected into the nasal side of the conjunctiva was completely drained, but a significant amount of the tracer injected into the temporal side largely remained in the conjunctiva (Figure 6, A-G). Moreover, we verified that the injected tracer (fluorescently labeled dextran, $\mathrm{MW}=3 \mathrm{kDa}$ ) was immediately drained through the conjunctiva lymphatics (Figure 6, H-M). Together, this study demonstrates differential drainage rates between the temporal and nasal sides, consistent with the polarized distribution of the conjunctiva lymphatics.

Pathological ocular lymphangiogenesis in the eyes of newborn pups. We noted that the limbal lymphatics give out multiple thin sprouts toward the cornea and that these thin lymphatic sprouts form mainly from the nasal-side limbal lymphatics but rarely from the temporal-side limbal lymphatics in the adult mouse eye (Figure 7, A-C). These short, blunt-ended lymphatic sprouts from the nasal-side limbal lymphatics seemed to be poised to respond to potential infection, playing a potential role in the ocular immune response. Consistent with these patterns, we detected a case of pathological corneal lymphangiogenesis at P7 in a rat pup, where an array of the limbal lymphatic sprouts grew toward the center of the avascular cornea (Figure 7D). Perinatal eye infection is a common eye complication in newborn humans and animals. Remarkably, the corneal lymphangiogenesis occurred mainly from the nasal side of the limbal lymphatics, whereas no sprouts were detected from the temporal side (Figure 7, E and F). Strikingly, Schlemm's canal remained unresponsive. Moreover, we detected another case of corneal pathological lymphangiogenesis in perinatal Prox1-EGFP newborn mice (P0.5), which occurred before the normal development of the ocular lymphatics and displayed irregular invasion into the center of the cornea (Figure 7G). Notably, the vascular growth patterns of developmental versus pathological ocular lymphangiogenesis were significantly different. While developmental lymphangiogenesis generated an organized, stepwise, and fractal lymphatic vessel growth from the nasal side of the eye (Figure $7 \mathrm{H})$, pathological lymphangiogenesis formed a nonfractal, irregular lymphatic network that initiated from a separate lymphatic vascular root (Figure 7I). 

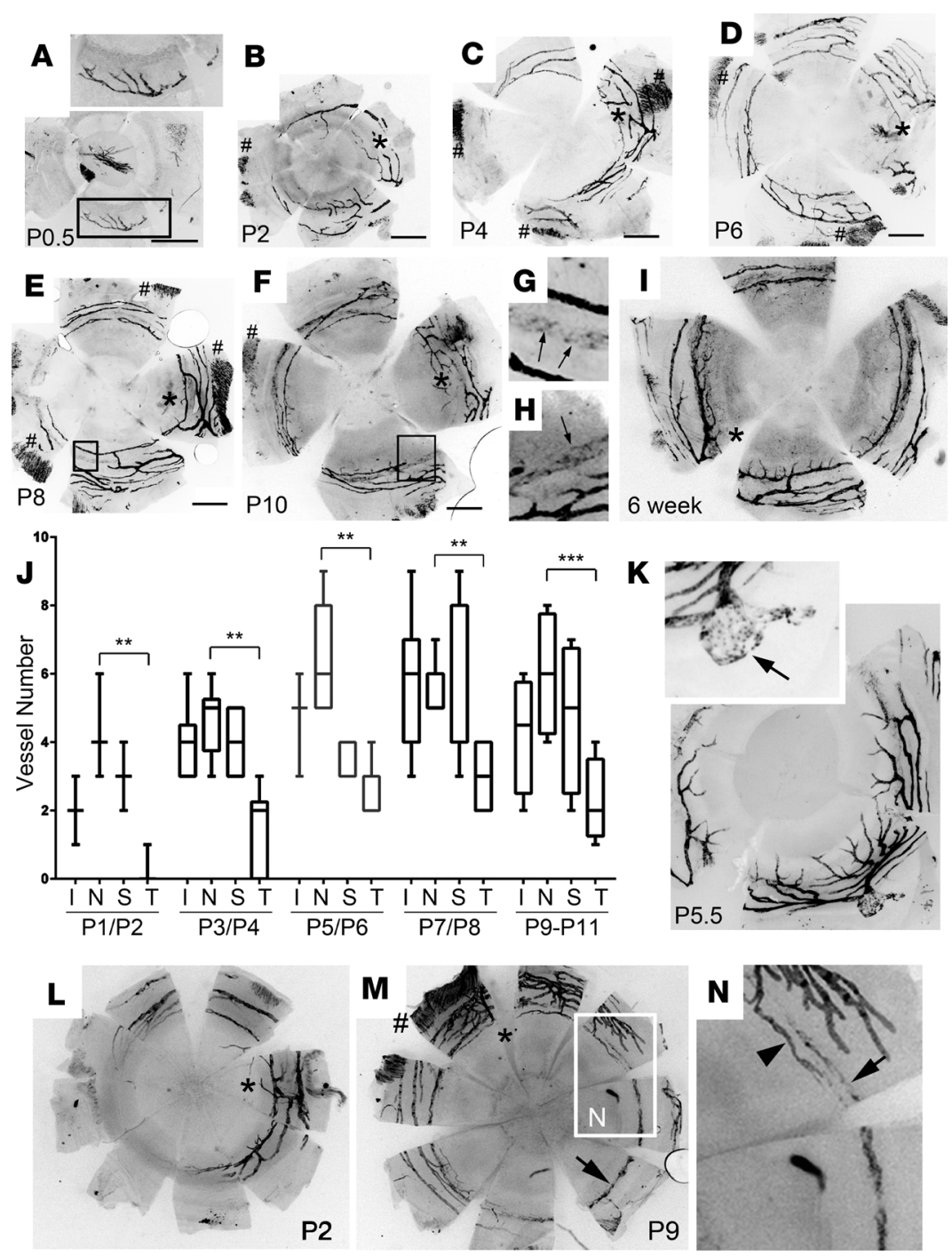

Figure 4. Postnatal development of the ocular lymphatics originating from the nasal side in mice. (A-I) Images showing the sequential lymphatic growth in the conjunctiva and limbus. The eyes from Prox1 reporter pups at P0.5, P2, P4, P6, P8, and P10, and 6-week-old adults, were enucleated, and cornea flat mounts were prepared. Asterisks point to the nasal side of the eye, and pound signs label the ocular muscles for the expression of Prox 1 in ocular muscles $(7,39)$. Note that the ocular lymphatics originate from the nasal side and grow into the limbus (inner circle) and conjunctiva (outer circles). The formation of Schlemm's canal (arrows) is evident by P8, and the boxed area in panels $\mathbf{E}$ and $\mathbf{F}$ are enlarged in panels $\mathbf{C}$ and $\mathbf{H}$, respectively. Scale bars: $0.5 \mathrm{~mm}$. Per time point, 3-7 pups (both sexes) were used for each experiment, and 1 representative image is shown. (J) A box plot showing the quantitative analyses of lymphatic vessels encircling the cornea in the inferior (I), nasal (N), superior (S), and temporal (T) quadrants of the eyes at P1/P2 $(n=3)$, P3/P4 ( $n=6)$, P5/P6 $(n=7)$, P7/P8 $(n=7)$, and P9-P11 ( $n$ $=4$ ). The number of lymphatic vessels seems to decrease in some regions in the older pups. However, this is due to sampling variation without a statistical significance. Statistical significance ( $P$ value) was calculated using unpaired $t$ test ( 2 tailed); ${ }^{* *} P<0.01$, and ${ }^{* *} P<0.001$. (K) The entire ocular lymphatic vascular tree seems to be rooted in a major lymphatic trunk in the eye of a Prox1 reporter pup (P5.5). In the enlarged inset, an arrow points to the disconnected lymphatic trunk. (L-N) Cornea flat mounts prepared from Prox1-EGFP reporter rats at P2 (L) and $P 9(M)$ showing that the ocular lymphatics in rats also originate from the nasal side (asterisks). The boxed area is enlarged in panel $\mathbf{N}$ to show the limbal lymphatic vessels (arrowhead) growing around the cornea edge and Schlemm's canal (arrow) forming nearby. \#, ocular muscle. Original magnification, $\times 4.6(\mathbf{C}), \times 2.7(\mathbf{H}), \times 2.5$ (inset in $\mathbf{K})$, and $\times 3.7(\mathbf{N})$.

Comparative analyses of the postnatal ocular angiogenesis and lymphangiogenesis. Because the limbal and conjunctival area is also heavily endowed with blood vessels $(42,43)$, we next performed comparative analyses of the angiogenesis and lymphangiogenesis in the anterior segment of the eyes. We first stained the anterior region of the eyes from Prox1-tdTomato mouse pups (P4) against Cd31, a panendothelial cell marker that is highly expressed in blood vessels but only weakly in lymphatic vessels (44). While lymphatic vessels were
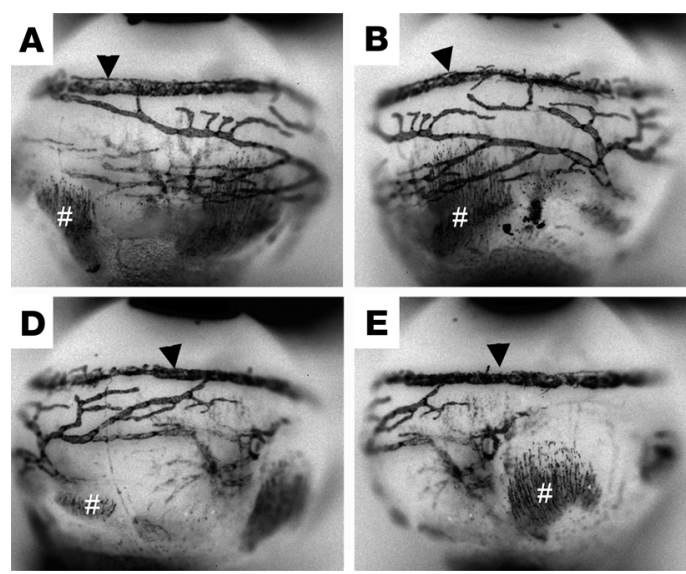
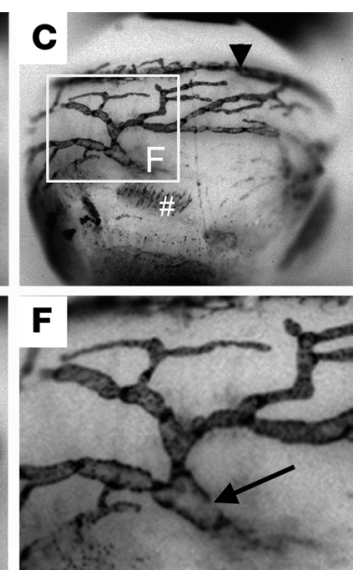

Figure 5. The conjunctival lymphatic vessels exit the eye through 1 collecting vessel. Lateral view of Prox1-tdTomato adult eye (8 weeks old). (A-E) Images were captured by rotating the eye counterclockwise. The boxed area is enlarged in panel $\mathbf{F}$ (original magnification, $\times 2.5$ ). Schlemm's canal (arrowheads), ocular muscles (\#), root collecting lymphatic vessel (arrow). 


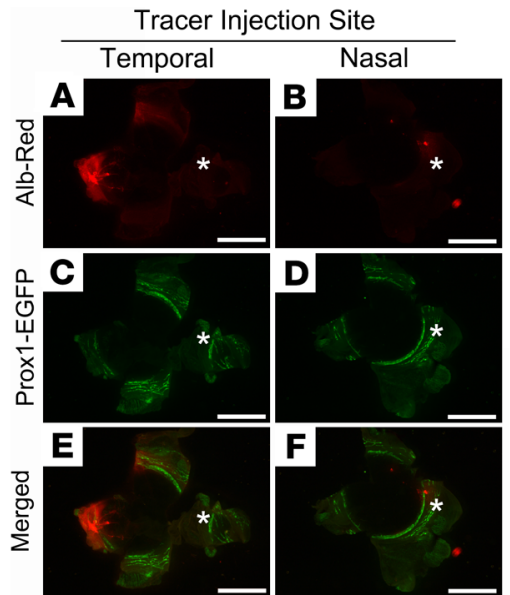

G

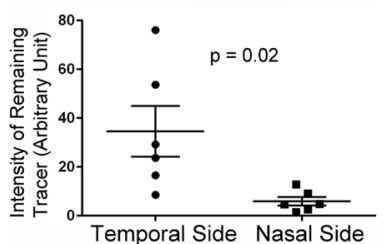

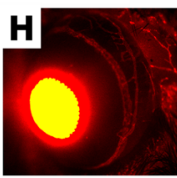
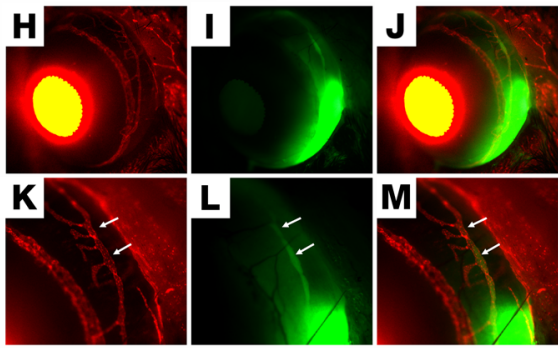

Figure 6. Faster fluid drainage in the nasal side compared with the temporal side. (A-G) A red fluorescent tracer (albumin red, $1 \mu \mathrm{L}$ ) was injected into the conjunctiva of the temporal side $(\mathbf{A}, \mathbf{C}$, and $\mathbf{E})$, or nasal side (B, D, and F) of the left eyes of anesthetized Prox1-EGFP mice. The same injection pressure was applied for all injections using a microinjector. After 10 minutes, the animals were euthanized, and their eyes were harvested to prepare the corneal flat mount. The remaining tracer in the cornea was quantified by measuring the intensity using NIH ImageJ software. (G) Graph showing the relative intensity of the remaining tracer in the temporal versus nasal side. Asterisks mark the nasal side. Scale bars: $2 \mathrm{~mm}$. The values are shown as mean \pm SEM. Statistical significance ( $P$ value) was calculated by unpaired $t$ test ( 2 tailed) on mice (both sexes). Six eyes were used for each group, and 12 mice were used for the experiment. Alb-Red, albumin red. (H-M) Images showing uptake and drainage of fluorescent tracer by conjunctival lymphatics. Dextran conjugated with fluorescein (Lysine Fixable, $0.5 \mu \mathrm{L}$ ) was injected into the temporal region of the eye of anesthetized Prox1-tdTomato mice. Immediately after injection, the mice were euthanized, and the eyelids and peripheral tissues excised. Fluorescence images were obtained from the intact eye globe without enucleation. (H and $\mathbf{K})$ Lymphatic networks, (I and $\mathbf{L}$ ) fluorescent tracer, and ( $\mathbf{~ a n d} \mathbf{M}$ ) merged images. Arrows mark the collecting lymphatic vessel carrying the tracer from the injection site. Four adult mice (both sexes) were used to yield a consistent result.

still growing and encircling the cornea, blood vessels had already covered the sclera, limbus, and iris at this developmental stage (Figure 8, A-C). Moreover, while the main lymphatic trunk was growing from the nasal side, the blood vessels had already entered the limbus area. This is consistent with a previous report that the limbus vasculature derives primarily from the anterior ciliary arteries and their branches form the vascular arcades that perfuse the limbal conjunctiva and peripheral cornea (45). We next employed the Flt1-tdsRed reporter mouse line (46), which marks BECs with a red fluorescent tdsRed protein. Flt1-tdsRed reporter was mated with the Prox1-EGFP line to produce double-transgenic mice (Flt1-tdsRed Prox1-EGFP) to visualize both blood and lymphatic vessels simultaneously. At P1, the limbus and conjunctiva were entirely covered with blood vessels, as previously shown (47), but the lymphatics initiated growth into the
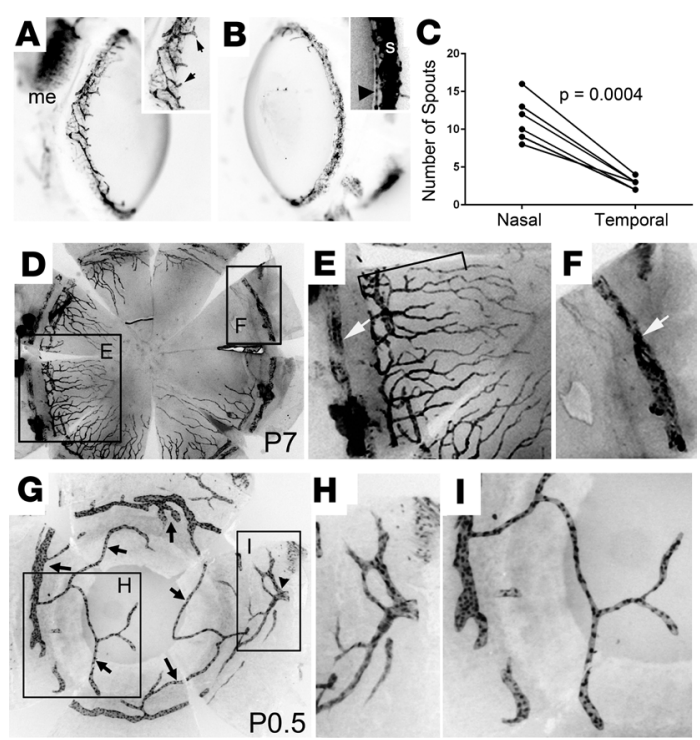

Figure 7. Pathological ocular lymphangiogenesis. (A-C) Short lymphatic sprouts are found in the nasal-side limbal lymphatics. Lateral views of the nasal side (A) and temporal side (B) of a healthy Prox1-tdTomato mouse eye (8 weeks) demonstrate the predominant presence of limbal lymphatic sprouts in the nasal side. A part of the nasal and temporal lymphatics was enlarged and shown in the insets. Black arrows, sprouts from the limbal lymphatic vessel; black arrowhead, the limbal lymphatic vessel; S, Schlemm's canal; me, medial rectus muscle. (C) The number of limbal lymphatic sprouts in the nasal versus temporal quadrant was quantified ( $n=6$, both sexes). (D-F) Extensive corneal lymphangiogenesis in the postnatal Prox1-EGFP rat eye (P7) due to unknown causes. White arrows point to Schlemm's canal, and a bracket marks active corneal lymphangiogenesis. Boxed areas in panel $\mathbf{D}$ are enlarged in panels $\mathbf{E}$ and $\mathbf{F}$, respectively. (G-I) Concurrent occurrence of developmental (arrowhead-marked) and pathological (arrow-marked) lymphangiogenesis detected in the eye of a Prox1-EGFP neonate mouse (P0.5). Boxed areas in panel $\mathbf{G}$ are enlarged in panels $\mathbf{H}$ (original magnification, $\times 2$ ) and $\mathbf{I}$ (original magnification, $\times 2.2$ ), respectively. 

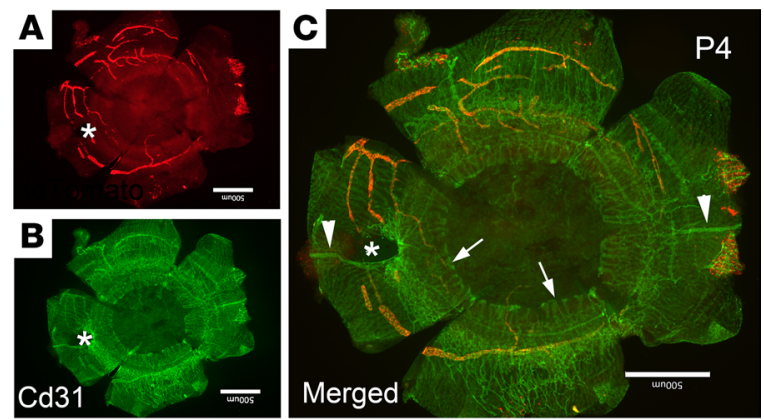

$\mathrm{BF}$

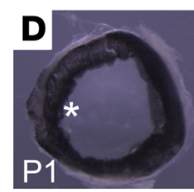

Flt1-tdsRed
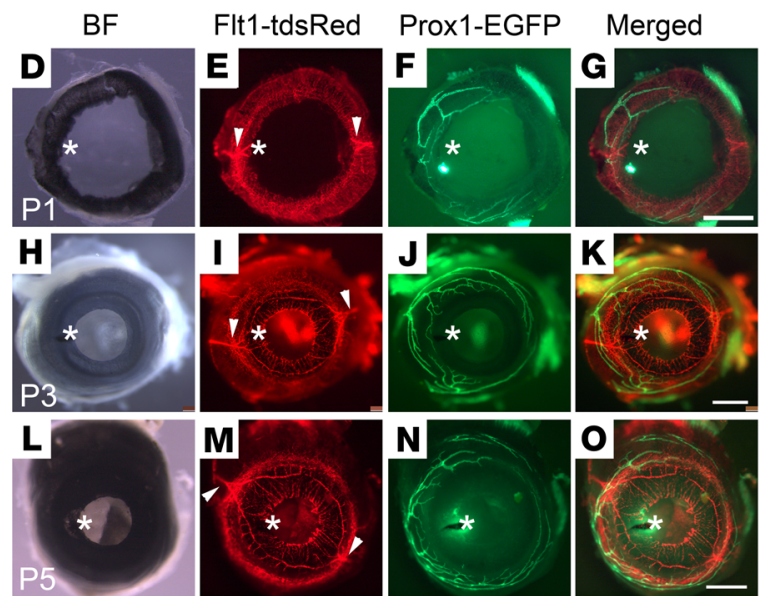

Figure 8. Postnatal development of blood and lymphatic vessels in the anterior eye. (A-C) A flat corneal mount of the eye of a Prox1-tdTomato reporter pup (P4) was stained for $[d 31$. Lymphatic vessels (tdTomato) (A) and Cd31-stained vessels (green) (B) revealed distinct and independent development of the 2 vasculatures in the anterior segment of the eye. (C) Merged image. (D-0) Front views of the anterior eyes of Flt1-tdsRed Prox1-EGFP double-transgenic pups revealed characteristic development of the ocular blood and lymphatic vessels at P1 (D-G), P3 (H-K), and P5 (L-0). Asterisk marks the nasal side. Arrowheads indicate long ciliary arteries, and arrows point to the iris. Scale bars: $500 \mu \mathrm{m}$. BF, bright-field.

limbal area only (Figure 8, D-G). At P3 and P5, blood vessels were evenly distributed, while the lymphatic vessels were still encircling the cornea toward the temporal side (Figure 8, H-O). Together, our study demonstrates that blood vessel development precedes lymphatic vessel development in the anterior region of the eyes and that both vasculatures independently develop without any significant spatiotemporal overlaps.

Polarized distribution of lymphatic vessels in human conjunctiva. We next asked whether the human eyes also display the similar polarized distribution of the conjunctiva lymphatics. To address this question, we obtained human cornea rims remaining after corneal transplantation and performed histological analyses on the perilimbal conjunctiva. All examined cornea rims retained an intact corneal-scleral junction and a skirt of the bulbar conjunctiva. Unfortunately, the nasal-temporal orientation information was not available for these specimens. Thus, we aimed to verify whether the human eyes displayed the similar patterns of the lymphatic and blood vessel distributions as observed in our rodent models. To assess the vascular distribution, we segmented the cornea rims into 6 equally sized fragments, embedded them in 1 paraffin block cassette, prepared their cross sections, stained for CD31 and LYVE1, and quantified the vessel numbers. Indeed, anti-LYVE1 staining revealed a polarized distribution of lymphatic vessels (i.e., vessel number) in the conjunctival area, whereas anti-CD31 staining revealed largely even vascular distribution (Figure 9, A-G). Therefore, this finding from the human eye rims is in agreement with the observations that we made in rodent eyes.

\section{Discussion}

In this study, we investigated the development and function of the ocular lymphatics and Schlemm's canal. Our results highlighted substantial differences in morphological structures, development timeline, and vessel distribution patterns between ocular lymphangiogenesis and Schlemm's canal formation using 3 independent lymphatic reporter animals. Notably, we illustrated a stepwise process of the ocular lymphatic network formation (Figure 10). The ocular lymphangiogenesis begins with nascent lymphatic vessels emerging from the nasal side of the developing eye. These rapidly growing lymphatics sprout, bifurcate, and quickly encircle the cornea in both a clockwise and counterclockwise direction toward the temporal side of the eye. During this developmental window, limbal and conjunctival lymphatics make frequent connections between them until they cover the entire conjunctival area. The nasal side is more enriched with lymphatics compared with the temporal side, presumably due to the entrance pattern of the major lymphatic trunk from the medial canthus region. In addition, more lymphatic sprouts are also produced by the limbal lymphatics in the nasal side. The polarized ocular lymphatics raised the possibility that fluid drainage and immune surveillance may be more efficient in the nasal side than the temporal side of the eye. Indeed, we found that the nasal side was more efficient in tracer drainage and more responsive to postnatal infection compared with the temporal side of the eye. In contrast, the blood vessels in the anterior surface of the eyes did not display this kind of polarized vascular pattern. Importantly, this polarized predilection of the ocular lymphatics was also conserved in human eyes.

The outcomes of our study provide important considerations for glaucoma surgeries, which aim to lower the IOP. Glaucoma surgeries, including trabeculectomy, generally create new passages that allow rerouting of the aqueous humor from the anterior chamber. As a result, rerouted fluid accumulates and forms a small reservoir, also called a bleb, at the subconjunctival space until the fluid is drained. Interestingly, it has been proposed that proper posttrabeculectomy fluid drainage and IOP management are heavily dependent on the function of the ocular lymphatic system and its association with the formed blebs $(48,49)$. 


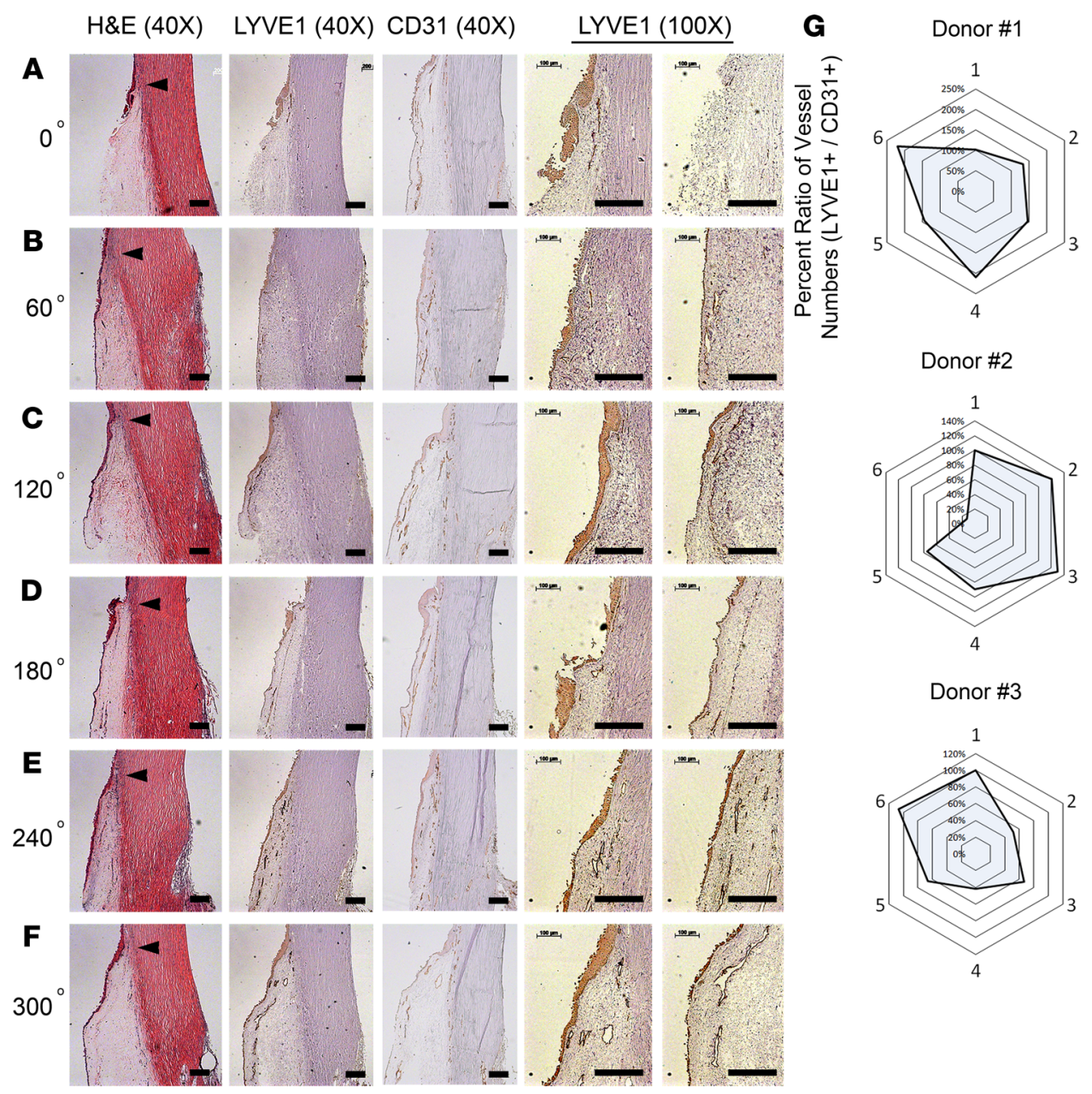

Figure 9. Distribution of conjunctival blood and lymphatic vessels in the anterior part of the human eye. (A-F) Cornea rim tissues, remaining after excision of the cornea for transplantation, were equally divided into 6 segments $60^{\circ}$ apart from each other, paraffin-embedded, and cross-sectioned for immunohistochemistry for H\&E, LYVE1, and CD31 images (original magnification, $\times 40$ ) and for LYVE1 images (original magnification, $\times 100$ ). Segment 1 was randomly chosen and labeled as $0^{\circ}$ (or $360^{\circ}$ ) segment. Arrowheads point to the cornea-conjunctiva junctions. Scale bars: $200 \mu \mathrm{m}$. (C) Hexagon chart showing the percentage ratio of $L Y V E 1^{+}$to $\mathrm{CD} 1^{+}$vessel number in each segment. In each segment, LYVE1 ${ }^{+}$vessel number was divided by $\mathrm{CD} 31^{+}$vessel number, which was then converted to a percentage in reference to the ratio in segment $1(100 \%)$. Segment numbers are shown at the corners of the hexagon. Three human donor eye rims were analyzed. Images shown in panels $\mathbf{A}-\mathbf{F}$ were from donor 1.

Based on tracer studies, trabeculectomy blebs showing a clear lymphatic outflow have been correlated with better IOP-lowering results $(48,49)$. An exciting implication these studies raised was that Mitomycin C, routinely applied on the surgery site to limit tissue scarring that would block newly created passages, could inhibit the growth of these draining lymphatics and potentially have a negative effect on the outcome of the surgery. In agreement with this notion, a previous study found that failed blebs previously treated with Mitomycin C resulted in decreased lymphatic and blood vessel density (21). Thus, healthy ocular lymphatics may play a major role in fluid clearance in bleb-forming glaucoma surgeries and the resulting IOP reduction. As such, the conjunctiva lymphatics were postulated to play a critical role in draining the bleb fluid $(50,51)$. Currently, trabeculectomy blebs are often placed in the superior or temporal quadrants to minimize potential postsurgery infections and to be hidden by the eyelid. Since our study uncovered the nasal-side predilection of the conjunctival lymphatics, a nasal or superior-nasal placement of the bleb may serve as alternative surgery sites for better outflow drainage and infection management.
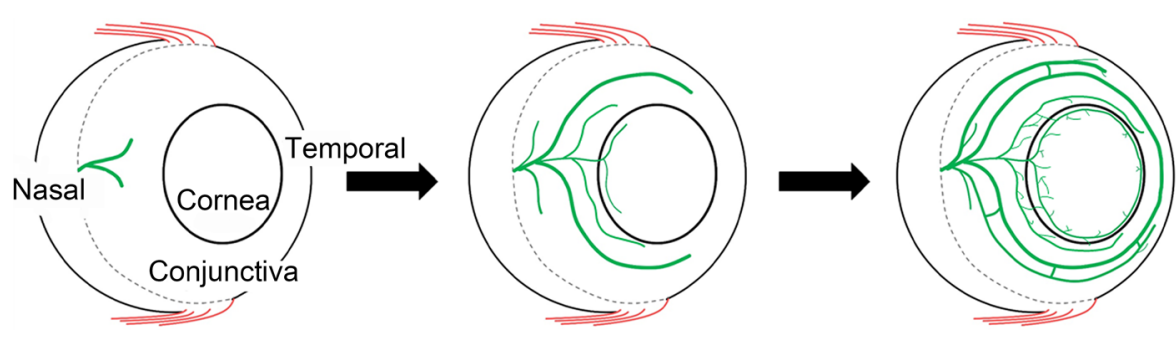

Figure 10. A simplified diagram illustrating the development and distribution of the postnatal limbal and conjunctival lymphatics. 
In addition, our new understanding of the polarized vascular pattern of the ocular lymphatics may be clinically significant for drug delivery as well. Subconjunctival space often acts as an important reservoir for iatrogenically injected subconjunctival medicines, such as antibiotics and steroids, for ocular disease treatment through transscleral delivery. Drugs injected into the subconjunctival space also form another type of bleb. The injected drug will be either delivered through the sclera to the targets or wastefully cleared by the conjunctival lymphatics. Thus, the presence of abundant lymphatics near the bleb could negatively affect the delivery of the drugs, as these drugs could be rapidly drained and cleared by the conjunctival lymphatic outflow. For example, temporal injection of the recently developed liposomal latanoprost $(52,53)$ will lead to sustained IOP lowering and reduction in drug clearance. The anatomical differences in administering the drugs subconjunctivally have not been determined. This would prove an essential aspect to achieve an optimal therapeutic effect if lymphatic drainage is to be considered a factor. Therefore, avoiding the lymphatic-rich region of the eye by injecting drugs on the temporal side may enhance the drug's longevity and subsequent delivery through the sclera into the eye, as opposed to leaving the eye via lymphatic drainage.

In conclusion, our work demonstrates the spatiotemporal development and polarized distribution of the ocular surface lymphatics to the nasal side of the eye in mice and rats. Consistent with these data, our human data support the nasal predilection of the ocular surface lymphatics. This may have clinical relevance for glaucoma treatment and drug delivery, which can significantly affect all aspects of eye care. Future work can be guided by developmental results and directed at identifying pharmacological/molecular tools to manipulate lymphatic presence (either greater or lesser) to aid in the treatment of eye diseases.

\section{Methods}

Animal-related studies. Mice and rats were housed and raised in air-filtered, clear cages in a 12-hour light/12hour dark cycle environment and fed ad libitum. The Flt1-tdsRed BAC transgenic mouse (46), Prox1-EGFP BAC transgenic mouse (39), Prox1-tdTomato BAC transgenic mouse (38), and Prox1-EGFP BAC transgenic rat (7) were previously reported. All mice were maintained in mixed backgrounds. Prox1-EGFP rats were maintained in the Sprague-Dawley outbred background. Both sexes were used for all experiments. Adult mice/rats were between 6 and 8 weeks old, and the ages of the pups are specified in figures or legends.

Corneal flat mount preparation. Corneal flat mounts were prepared as previously described (54). Briefly, mice and rats were euthanized by carbon dioxide asphyxiation followed by cervical dislocation or thoracic puncture, respectively. A marking hole was made on the nasal side of the eye using a syringe needle as a future directional reference. The eyes were then enucleated and fixed in $4 \%$ paraformaldehyde (PFA) at $4^{\circ} \mathrm{C}$ for $2-4$ hours. Fixed eyes were cut in half, and the anterior half, including the cornea, limbus, and conjunctiva, was then flattened on slides as previously described (54). All images were captured using a Leica Stereomicroscope (Leica M165 FC) or a Zeiss ApoTome Microscope (Zeiss AxioVision). Some fluorescent images were gray-scaled and then inverted to black-and-white images to increase their clarity. Anti-Cd31 (BD Biosciences, 553370), anti-Lyve1 (Angiobio, 11-034), and anti-Vegfr3 (R\&D Systems, Bio-Techne, AF743) antibodies were used for immunofluorescence staining

Tracer injection assay. Adult Prox1-EGFP mice (males and females, $6-8$ weeks, total $n=6$ /group) were anesthetized by isoflurane. One microliter $(\mu \mathrm{L})$ of bovine serum albumin, Alexa Fluor 594 conjugate (Thermo Fisher Scientific), was injected into the conjunctival space of the temporal or nasal side of the left eye using a syringe controlled by a microinjection apparatus (World Precision Instruments) under the same syringe pump pressure. After 10 minutes postinjection, mice were euthanized, and the eyes were enucleated. Corneal flat mounts were prepared, and the images were captured by a fluorescence stereomicroscope. The amount of the remaining tracer was quantified using NIH ImageJ. For the conjunctival lymphatic uptake study, $0.5 \mu \mathrm{L}$ of fluorescein-conjugated dextran (Lysine Fixable dye, Thermo Fisher Scientific) was injected into the temporal side of the anesthetized Prox1-tdTomato mouse ( $n=4$, both sexes). Immediately after the injection of the dye, the mice were euthanized, and eyelids and peripheral tissues were excised. Fluorescence images were obtained from the intact eye globe.

Human tissue studies, histology, and morphometric analyses. Tissues were fixed either in 4\% PFA overnight to make frozen sections or in formalin for 48 hours to prepare paraffin sections. Immunofluorescence or immunohistochemistry assays were performed using anti-LYVE1 (R\&D Systems, Bio-Techne, AF2089) or anti-CD31 (Dako, M0823) antibodies. Vascular area and numbers were measured from more than 5 representative images per group using NIH ImageJ software. Three deidentified human cornea rims were collected and analyzed. 
Statistics. No statistical method was used to predetermine the sample size. The values are presented as mean \pm SEM. Statistical differences between the experimental and control groups were determined by 2-tailed $t$ test (either unpaired or paired) using Microsoft Excel and GraphPad Prism 6 (GraphPad Software, Inc.). P values less than 0.05 were considered statistically significant. Box-and-whisker plots were drawn using GraphPad Prism 6 .

Study approval. Approval of the animal-based studies was obtained from the Institutional Animal Care and Use Committees at the USC. Deidentified human cornea rims were obtained after the excision of the cornea for transplantation under the approval of the Institutional Review Board of the USC.

\section{Author contributions}

ITC, AKW, MH, SZN, JCT, TTW, ASH, and YKH contributed research design. YW, YJS, KL, DC, EP, GHD, EJ, KB, LZ, SM, SD, PD, and DC conducted experiments, acquired data, and/or analyzed data. $\mathrm{MH}$ and ME provided reagents. YJS, ITC, AKW, MH, SZN, JCT, TTW, ASH, and YKH contributed to writing and editing of the manuscript.

\section{Acknowledgments}

This study was supported by the NIH (EY026260, HL121036, HL141857, DE027891, DK114645 to YKH; K08EY024674 and R01EY030501 to ASH; K08 HL132110 to AKW), Research to Prevent Blindness (Career Development Award to ASH), Glaucoma Research Foundation (Shaffer Grant to ASH), and American Cancer Center (IRG-16-181-57 to DC).

Address correspondence to: Young-Kwon Hong, Department of Surgery, University of Southern California, Norris Comprehensive Cancer Center, 1450 Biggy Street NRT6501, Los Angeles, California 90033, USA. Phone: 323.442.7825; Email: young.hong@usc.edu.

1. Scallan JP, Zawieja SD, Castorena-Gonzalez JA, Davis MJ. Lymphatic pumping: mechanics, mechanisms and malfunction J Physiol (Lond). 2016;594(20):5749-5768.

2. Breslin JW, Yang Y, Scallan JP, Sweat RS, Adderley SP, Murfee WL. Lymphatic vessel network structure and physiology. Compr Physiol. 2018;9(1):207-299.

3. Aspelund A, et al. The Schlemm's canal is a VEGF-C/VEGFR-3-responsive lymphatic-like vessel. J Clin Invest. 2014;124(9):3975-3986.

4. Kizhatil K, Ryan M, Marchant JK, Henrich S, John SW. Schlemm's canal is a unique vessel with a combination of blood vascular and lymphatic phenotypes that forms by a novel developmental process. PLoS Biol. 2014;12(7):e1001912.

5. Park DY, et al. Lymphatic regulator PROX1 determines Schlemm's canal integrity and identity. JClin Invest. 2014;124(9):3960-3974

6. Truong TN, Li H, Hong YK, Chen L. Novel characterization and live imaging of Schlemm's canal expressing Prox-1. PLoS One. 2014;9(5):e98245.

7. Jung E, et al. Development and characterization of a novel Prox1-EGFP lymphatic and Schlemm's canal reporter rat. Sci Rep. 2017;7(1):5577.

8. Wigle JT, Oliver G. Prox1 function is required for the development of the murine lymphatic system. Cell. 1999;98(6):769-778

9. Wigle JT, et al. An essential role for Prox 1 in the induction of the lymphatic endothelial cell phenotype. EMBO J. 2002;21(7):1505-1513.

10. Chauhan SK, Dohlman TH, Dana R. Corneal lymphatics: role in ocular inflammation as inducer and responder of adaptive immunity. J Clin Cell Immunol. 2014;5:1000256.

11. Yücel YH, et al. Identification of lymphatics in the ciliary body of the human eye: a novel "uveolymphatic" outflow pathway. Exp Eye Res. 2009;89(5):810-819.

12. Ji YW, et al. Corneal lymphangiogenesis facilitates ocular surface inflammation and cell trafficking in dry eye disease. Ocul Surf. 2018;16(3):306-313.

13. Yücel YH, et al. Active lymphatic drainage from the eye measured by noninvasive photoacoustic imaging of near-infrared nanoparticles. Invest Ophthalmol Vis Sci. 2018;59(7):2699-2707.

14. Notara M, Lentzsch A, Coroneo M, Cursiefen C. The role of limbal epithelial stem cells in regulating corneal (lymph)angiogenic privilege and the micromilieu of the limbal niche following UV exposure. Stem Cells Int. 2018;2018:8620172.

15. Yang JF, et al. Understanding lymphangiogenesis in knockout models, the cornea, and ocular diseases for the development of therapeutic interventions. Surv Ophthalmol. 2016;61(3):272-296.

16. Lee HS, et al. Involvement of corneal lymphangiogenesis in a mouse model of allergic eye disease. Invest Ophthalmol Vis Sci. 2015;56(5):3140-3148.

17. Sanchez I, Martin R. Advances in diagnostic applications for monitoring intraocular pressure in glaucoma: a review. J Optom. 2019;12(4):211-221.

18. Gabai A, Cimarosti R, Battistella C, Isola M, Lanzetta P. Efficacy and safety of trabeculectomy versus nonpenetrating surgeries in open-angle glaucoma: a meta-analysis. J Glaucoma. 2019;28(9):823-833.

19. Watanabe Y, Hamanaka T, Takemura T, Murakami A. Involvement of platelet coagulation and inflammation in the endothelium of Schlemm's canal. Invest Ophthalmol Vis Sci. 2010;51(1):277-283. 
20. Ramos RF, Hoying JB, Witte MH, Daniel Stamer W. Schlemm's canal endothelia, lymphatic, or blood vasculature? J Glaucoma. 2007;16(4):391-405

21. Bouhenni RA, et al. Lymphatic and blood vessel density in human conjunctiva after glaucoma filtration surgery. $J$ Glaucoma. 2016;25(1):e35-e38.

22. Esfandiari $\mathrm{H}$, et al. Long-term surgical outcomes of ab externo trabeculotomy in the management of primary congenital glaucoma. J AAPOS. 2019;23(4):222.e1-222.e5.

23. Chen L, Cursiefen C, Barabino S, Zhang Q, Dana MR. Novel expression and characterization of lymphatic vessel endothelial hyaluronate receptor 1 (LYVE-1) by conjunctival cells. Invest Ophthalmol Vis Sci. 2005;46(12):4536-4540.

24. Cursiefen C, et al. Inhibition of hemangiogenesis and lymphangiogenesis after normal-risk corneal transplantation by neutralizing VEGF promotes graft survival. Invest Ophthalmol Vis Sci. 2004;45(8):2666-2673.

25. Ecoiffier T, Yuen D, Chen L. Differential distribution of blood and lymphatic vessels in the murine cornea. Invest Ophthalmol Vis Sci. 2010;51(5):2436-2440.

26. Kang GJ, Truong T, Huang E, Su V, Ge S, Chen L. Integrin alpha 9 blockade suppresses lymphatic valve formation and promotes transplant survival. Invest Ophthalmol Vis Sci. 2016;57(14):5935-5939.

27. Schroedl F, et al. Consensus statement on the immunohistochemical detection of ocular lymphatic vessels. Invest Ophthalmol Vis Sci. 2014;55(10):6440-6442.

28. Zhang H, Hu X, Tse J, Tilahun F, Qiu M, Chen L. Spontaneous lymphatic vessel formation and regression in the murine cornea. Invest Ophthalmol Vis Sci. 2011;52(1):334-338.

29. Zhang H, et al. Novel discovery of LYVE-1 expression in the hyaloid vascular system. Invest Ophthalmol Vis Sci. 2010;51(12):6157-6161.

30. Zhu J, et al. Simultaneous in vivo imaging of blood and lymphatic vessel growth in Prox1-GFP/Flk1::myr-mCherry mice. FEBS J. 2015;282(8):1458-1467

31. Doh SJ, et al. Fluorescent reporter transgenic mice for in vivo live imaging of angiogenesis and lymphangiogenesis. Angiogenesis 2018;21(4):677-698

32. Le VNH, Hou Y, Horstmann J, Bock F, Cursiefen C. Novel method to detect corneal lymphatic vessels in vivo by intrastromal injection of fluorescein. Cornea. 2018;37(2):267-271.

33. Romano V, Steger B, Zheng Y, Ahmad S, Willoughby CE, Kaye SB. Angiographic and in vivo confocal microscopic characterization of human corneal blood and presumed lymphatic neovascularization: a pilot study. Cornea. 2015;34(11):1459-1465

34. Horstmann J, et al. Label-free in vivo imaging of corneal lymphatic vessels using microscopic optical coherence tomography. Invest Ophthalmol Vis Sci. 2017;58(13):5880-5886.

35. Grüntzig J, Djajadisastra A, Uthoff D, Mehlhorn H. [Fine structure of conjunctival lymphatic vessel valves]. Klin Monbl Augenheilkd. 1994;205(3):147-155

36. Zhong W, et al. Prox1-GFP/Flt1-DsRed transgenic mice: an animal model for simultaneous live imaging of angiogenesis and lymphangiogenesis. Angiogenesis. 2017;20(4):581-598.

37. Ecoiffier T, Sadovnikova A, Yuen D, Chen L. Conjunctival lymphatic response to corneal inflammation in mice. J Ophthalmol. 2012;2012:953187.

38. Hong M, et al. Efficient assessment of developmental, surgical and pathological lymphangiogenesis using a lymphatic reporter mouse and its embryonic stem cells. PLoS One. 2016;11(6):e0157126.

39. Choi I, et al. Visualization of lymphatic vessels by Prox1-promoter directed GFP reporter in a bacterial artificial chromosome-based transgenic mouse. Blood. 2011;117(1):362-365.

40. Sabine A, et al. Mechanotransduction, PROX1, and FOXC2 cooperate to control connexin 37 and calcineurin during lymphatic-valve formation. Dev Cell. 2012;22(2):430-445.

41. Jiang X, Nicolls MR, Tian W, Rockson SG. Lymphatic dysfunction, leukotrienes, and lymphedema. Annu Rev Physiol. 2018;80:49-70.

42. Chan CK, et al. Mouse strain-dependent heterogeneity of resting limbal vasculature. Invest Ophthalmol Vis Sci. 2004;45(2):441-447.

43. Nakao S, et al. Lymphangiogenesis and angiogenesis: concurrence and/or dependence? Studies in inbred mouse strains. FASEB J. 2010;24(2):504-513.

44. Baluk P, et al. Functionally specialized junctions between endothelial cells of lymphatic vessels. J Exp Med. 2007;204(10):2349-2362

45. Van Buskirk EM. The anatomy of the limbus. Eye (Lond). 1989;3(pt 2):101-108.

46. Matsumoto K, et al. Study of normal and pathological blood vessel morphogenesis in Flt1-tdsRed BAC Tg mice. Genesis. 2012;50(7):561-571

47. Seo S, et al. Forkhead box transcription factor FoxC1 preserves corneal transparency by regulating vascular growth. Proc Natl Acad Sci U S A. 2012;109(6):2015-2020.

48. Khoo YJ, Abdullah AAH, Yu DY, Morgan WH. Use of trypan blue to assess lymphatic function following trabeculectomy. Clin Experiment Ophthalmol. 2019;47(7):892-897.

49. Singh D. Conjunctival lymphatic system. J Cataract Refract Surg. 2003;29(4):632-633.

50. Collin HB. Endothelial cell lined lymphatics in the vascularized rabbit cornea. Invest Ophthalmol. 1966;5(4):337-354

51. Freeman LE, Troutt HF. Lymph drainage of the conjunctiva: topographic anatomic study in calves. Am J Vet Res. 1985;46(9):1967-1970.

52. Wong TT, Novack GD, Natarajan JV, Ho CL, Htoon HM, Venkatraman SS. Nanomedicine for glaucoma: sustained release latanoprost offers a new therapeutic option with substantial benefits over eyedrops. Drug Deliv Transl Res. 2014;4(4):303-309.

53. Natarajan JV, et al. Sustained drug release in nanomedicine: a long-acting nanocarrier-based formulation for glaucoma. ACS Nano. 2014;8(1):419-429.

54. Kim J, et al. Impaired angiopoietin/Tie2 signaling compromises Schlemm's canal integrity and induces glaucoma. J Clin Invest. 2017;127(10):3877-3896. 